\title{
Micromechanical Modeling of the Arterial Wall: Influence of Mechanical Heterogeneities on the Wall Stress Distribution and the Peak Wall Stress
}

\author{
M. Toungara*†, L. Orgéas $\dagger$, C. Geindreau† and L. Bailly $\ddagger$ \\ $†$ CNRS/University of Grenoble, Laboratoire 3SR, BP 53, 38041 Grenoble Cedex 9, France \\ † CNRS, Aix-Marseille University, IRPHE, 13384, Marseille, France
}

Keywords: Abdominal Aortic Aneurysm; wall stress; anisotropy; heterogeneous mechanical properties

\section{Introduction}

Previous tensile tests have shown the high anisotropy and heterogeneity of the arterial wall mechanical properties as a function of age, pathology (e.g. Abdominal Aortic Aneurysm, AAA) and location (Vande Geest et al, 2006). The issues of this anisotropic behavior and the AAA geometrical shapes have been tackled in many numerical studies. However, the wall mechanical heterogeneity has been very sparsely considered (Tierney et al, 2012). This work aims to study the effects of such heterogeneity on the stress distribution and the peak wall stress during a static pressurization. Thence, a micromechanical-based model was used for the wall and finite element analyses (FEA) were performed on idealized AAAs. Unlike many previous phenomenological models, the current constitutive model depends on five material parameters only, which also allow to control the wall microarchitecture.

\section{Methods}

Micromechanical model: The arterial wall was idealized as two lattices (I \& II), each comprising two families of straight fibers, embedded in an incompressible Neo-Hookean soft membrane. The lattice (Fig. 1.a) can be seen as a repetition of a Representative Elementary Cell (REC) as sketched in Fig. 1.b. Let us note $\mathbf{p}_{o i}=\ell_{0 i} \mathbf{E}_{i}$ and $\mathbf{p}_{i}=\ell_{i} \mathbf{e}_{i}$, the fibers vectors in the initial $\left(\mathcal{R}_{0}\right)$ and deformed $(\mathcal{R})$ configurations, respectively. In $\mathcal{R}_{0}$ (resp. in $\mathcal{R}$ ), the fiber $i$ has a length $\ell_{0 i}$ (resp. $\ell_{i}$ ) and is oriented along the unit vector $\mathbf{E}_{i}$ (resp. $\mathbf{e}_{i}$ ) making an angle $\theta_{0 i}^{I}\left(\operatorname{resp} . \theta_{i}^{I}\right)$ and $\theta_{0 i}^{I I}\left(\right.$ resp. $\left.\theta_{i}^{I I}\right)$ with the orthoradial direction $\mathbf{e}_{\theta}$ of the wall. The tension in a fiber $i$ is defined as: $\mathbf{t}_{i}=\mathrm{c}_{0}\left(\ell_{i} / \ell_{0 i}\right)\left\{\exp \left[\mathrm{c}_{1} / 2\left(\left(\ell_{i} / \ell_{0 i}\right)^{2}-1\right)\right]-1\right\} \mathbf{e}_{i}$, where $c_{0}$ and $c_{1}$ are material parameters. From the REC and its micromechanics, the homogenization provides the macroscopic Cauchy' stress tensor:

$$
\boldsymbol{\sigma}=\boldsymbol{\sigma}_{m}+\frac{1}{h_{0}\left\|\mathbf{p}_{1} \wedge \mathbf{p}_{2}\right\|} \sum_{i=1}^{2} \mathbf{t}_{i} \otimes \mathbf{p}_{i}+\frac{1}{h_{0}\left\|\mathbf{p}_{3} \wedge \mathbf{p}_{4}\right\|} \sum_{i=3}^{4} \mathbf{t}_{i} \otimes \mathbf{p}_{i}
$$

where $\boldsymbol{\sigma}_{m}$ is the stress tensor of the soft membrane and $h_{0}$ an arbitrary thickness. The second and the third terms in the right side correspond to the first (I) and the second (II) lattices (Bailly et al, 2012).

Microstructure optimization: Each microstructure parameter was adjusted with biaxial tensile data (Vande Geest et al, 2006). An optimization process based on a least-squares approach was used. The parameters identified on average data (26 AAA samples and 8 AA samples, mean age: $70 \pm 4$ ) are summarized in Table 1. Details of the discrepancies between experimental and optimized data are reported in Bailly et al (2012).

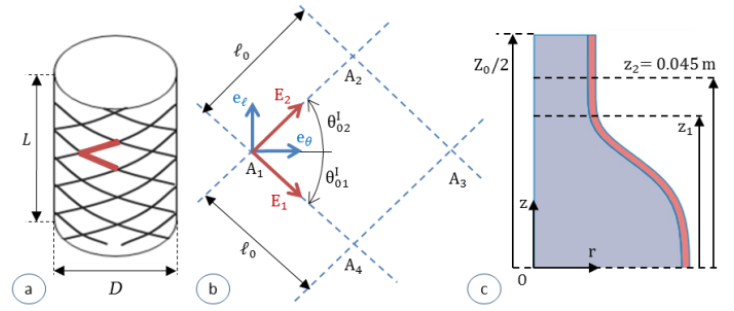

Figure 1 (a) Lattice I (b) In-plane view of the Representative Elementary Cell in $\mathcal{R}_{0} ;$ (c) Geometry of an axisymmetric AAA $\left(F_{e}=0\right)$. When $|z| \geq z_{2}$, the artery is assumed to be healthy; the artery total length $Z_{0}=0.1 \mathrm{~m}$

\begin{tabular}{|l|c|c|c|c|c|}
\hline & $\boldsymbol{c}_{\mathbf{0}}(\mathrm{N})$ & $\boldsymbol{c}_{\mathbf{1}}$ & $\boldsymbol{\theta}_{\mathbf{0}}^{\boldsymbol{I}}\left({ }^{\circ}\right)$ & $\boldsymbol{\theta}_{\mathbf{0}} \boldsymbol{I I}\left({ }^{\circ}\right)$ & $\boldsymbol{\ell}_{\mathbf{0}}(\mathrm{mm})$ \\
\hline AA & 0.00140 & 37.99 & 31 & 60 & 5.0 \\
\hline AAA & 0.00076 & 70.73 & 27 & 56 & 5.0 \\
Table 1 Model parameters for AA (healthy aorta) \\
and AAA tissues
\end{tabular}

SolidWorks ${ }^{\odot}$, using a mathematical form as in Toungara et al (2012). These AAAs were characterized by the dilatation parameter $\left(R_{a n} / R_{a}=2.75\right), \quad$ the eccentricity $\left(F_{e}=\right.$ $\left.e /\left(R_{a n}-R_{a}\right)\right)$ and the aspect ratio $\left(L_{a n} / R_{a n}=\right.$ $2.50)$, with $R_{a}=D / 2=10 \mathrm{~mm}$ the $\mathrm{AA}$ radius, $R_{a n}$ the AAA maximum radius and $L_{a n}$ the AAA length. The shift of $R_{a n}$ from the AA axis is defined by $e$. For sake of simplicity a uniform and constant wall thickness $(1.50 \mathrm{~mm})$ is adopted. Fig. 1.c shows an example of an axisymmetric AAA $\left(F_{e}=0\right)$.

Mechanical heterogeneity: Pathological and healthy material parameters were consider in the bulged $\left(0 \leq|z| \leq z_{1}\right)$ and the straight $\left(z_{2} \leq|z| \leq Z_{0} / 2\right)$ regions of the artery (see Fig. 1.c and Table 1), respectively. A linear evolution of these parameters was proposed in the AA-AAA transitional zone $\left(\mathrm{z}_{1}\right.$ $\left.\leq|\mathrm{z}| \leq \mathrm{z}_{2}\right)$. Thereafter, in the lack of experimental data, the width of this zone was parametrically varied: $z_{1} \in\left[0, z_{2}\right]$.

Numerical simulations: The above material model was implemented in Comsol Multiphysics ${ }^{\circledR}$ where FEA were performed. U-P formulation was adopted 
with Lagrangian P2-P1 finite elements. A typical structured mesh was adopted in this study. At both proximal and distal ends of the artery, the axial displacement was set to zero while the radial one was free. Finally, the mean systolic pressure (120 $\mathrm{mmHg}$ ) was applied at the inner side of the AAA.

\section{Results and Discussion}

The distribution and the peak of the first principal stress, $\sigma_{1}$ and $\sigma_{1 \mathrm{max}}$, were analyzed as a function of the AAA eccentricity $\left(F_{e}\right)$ and the width of the AA-AAA transitional zone.

Homogeneous AAA wall: When the AAA material parameters were adopted for the whole artery, $\sigma_{1 \mathrm{max}}$ is observed in the proximal / distal region of the artery (Fig. 2.a). This maximum is localized in the anterior region of the AAA when the latter is asymmetric $\left(F_{e} \neq 0\right)$. Moreover, $\sigma_{1 \max }$ increases with the increase of the eccentricity: $\sigma_{1 \text { max }}(\mathrm{kPa})=$ $\{655,876,986\}$ for $F_{e}=\{0.0,0.5,1.0\}$. In the same way, when the AA material parameters were considered for the whole artery, $\sigma_{1 \text { max }}(\mathrm{kPa})=\{719$, $965,1031\}$ for $F_{e}=\{0.0,0.5,1.0\}$, resulting in an increase of $5-10 \%$ in $\sigma_{1 \mathrm{max}}$. Previously, using a phenomenological material model for the AAA wall, we had obtained $\sigma_{1 \text { max }}=1655 \mathrm{kPa}$ for the most asymmetric AAA (Toungara et al, 2012). In that case, the material model was stiffer than the one used here. However, the value of $\sigma_{1 \text { max }}$ in the present study is still very close to the average ultimate stress (1019 $\mathrm{kPa})$ observed in uniaxial tensile tests on AAA tissues (Raghavan et al, 1996).

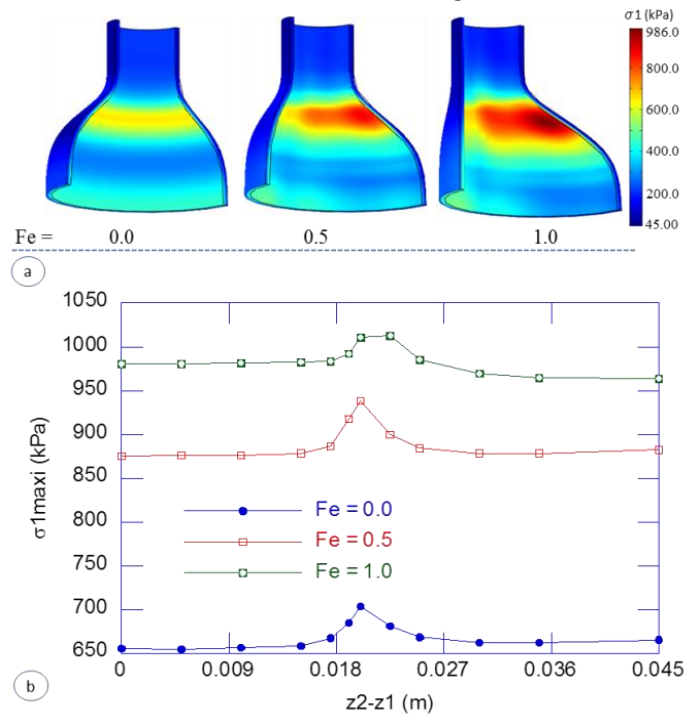

Figure 2 (a) First principal stress $\left(\sigma_{1}\right)$ distribution in homogeneous wall; (b) heterogeneous wall, influence of AA-AAA transitional zone on $\sigma_{1 \max }$ Heterogeneous AAA wall: When the AA and the AAA regions are considered with proper mechanical properties and with different widths $\left(\mathrm{z}_{2}\right.$ $z_{1}$ ) of the transitional zone, the previous stress distribution (Fig. 2.a) remains unchanged. But, from the homogeneous case, $\sigma_{1 \max }$ increases with about $7 \%$ when the limit of the pathological region of the artery $\left(z_{1}\right)$ is in the geometrical junction of the straight and the bulged parts (Fig. 1.c), i.e. for $\mathrm{Z}_{2}-\mathrm{Z}_{1} \approx 0.02 \mathrm{~m}$, whatever the AAA eccentricity (Fig. 2.b). Similar results were recently observed by Tierney et al (2012). These authors considered the wall mechanical heterogeneity in patient-specific AAAs, by dividing the wall into four regions (posterior, anterior, right and left laterals), each with its own material parameters. From the homogeneous case, an increase of about $10 \%$ in $\sigma_{1 \text { max }}$ was obtained with the heterogeneous wall.

\section{Conclusions}

Micromechanical-based anisotropic and heterogeneous model was proposed for the arterial tissue. This model was implemented in FEA software and the wall stress distribution and the peak wall stress were computed in idealized AAAs. The preliminary results are in agreement with previous ones from the literature. We also highlighted the prevalence of structural effects, versus material properties, namely the heterogeneity, on the numerical prediction of the peak wall stress. Therefore, when predicting the AAA rupture or manufacturing phantom AAAs, besides the wall mechanical properties, special caution should be paid to geometrical aspects. Finally, let us remark that the used micromechanical model permits to consider the fibers arrangement and their mechanical behavior. Consequently, more complex behavior for fibers (stiffening, damage...) can be readily introduced in order to predict the AAAs rupture.

\section{References}

Bailly L, Geindreau C, Orgéas L, Deplano V, 2012, Towards a Biomimetism of Abdominal Healthy and Aneurysmal Arterial Tissues, J. Mech. Beha. Biomed. Mater., 10:151-65

Raghavan M L, Webster M W, Vorp D A, 1996, Ex-Vivo Biomechanical Behavior of Abdominal Aortic Aneurysm: Assessment Using a New Mathematical Model, Ann. Biomed. Eng., 24:573-82

Tierney A P, Callanan A, McGloughlin T M, 2012, Use of Regional Mechanical Properties of Abdominal Aortic Aneurysms to Advance Finite Element Modeling of Rupture Risk, J. Endovasc. Ther., 19:100-14.

Toungara M, Chagnon G, Geindreau C, 2012, Numerical Analysis of the Wall Stress in Abdominal Aortic Aneurysm: Influence of the Material Model Near-Incompressibility, J. Mech. Med. Biol., 12:1250005-19

Vande Geest J P, Sacks M S, Vorp D A, 2006, The Effects of Aneurysm on the Biaxial Mechanical Behavior of Human Abdominal Aorta, $J$. Biomech., 39:1324-34 